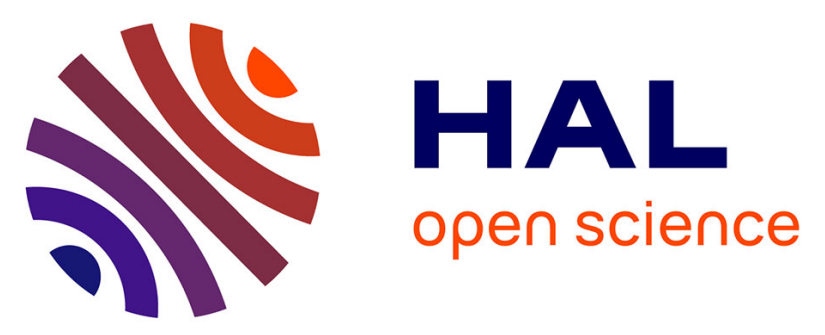

\title{
Clinical and anatomical predictors for freezing of gait and falls after subthalamic deep brain stimulation in Parkinson's disease patients
}

Carine Karachi, Florence Cormier-Dequaire, David Grabli, Brian Lau, Hayat Belaid, Soledad Navarro, Marie Vidailhet, Eric Bardinet, Sara

Fernandez-Vidal, Marie-Laure Welter

\section{To cite this version:}

Carine Karachi, Florence Cormier-Dequaire, David Grabli, Brian Lau, Hayat Belaid, et al.. Clinical and anatomical predictors for freezing of gait and falls after subthalamic deep brain stimulation in Parkinson's disease patients. Parkinsonism \& Related Disorders, 2019, 62, pp.91 - 97. 10.1016/j.parkreldis.2019.01.021 . hal-03485848

\section{HAL Id: hal-03485848 \\ https://hal.science/hal-03485848}

Submitted on 20 Dec 2021

HAL is a multi-disciplinary open access archive for the deposit and dissemination of scientific research documents, whether they are published or not. The documents may come from teaching and research institutions in France or abroad, or from public or private research centers.
L'archive ouverte pluridisciplinaire HAL, est destinée au dépôt et à la diffusion de documents scientifiques de niveau recherche, publiés ou non, émanant des établissements d'enseignement et de recherche français ou étrangers, des laboratoires publics ou privés.

\section{(ㅇ)(1) $\$$}

Distributed under a Creative Commons Attribution - NonCommerciall 4.0 International 


\section{Clinical and anatomical predictors for freezing of gait and falls after subthalamic deep brain stimulation in Parkinson's disease patients}

Carine Karachi, MD, PhD*, 1,2,3,4 Florence Cormier-Dequaire, MD*, 1,2,3,5 David Grabli, MD, PhD, ${ }^{1,2,3,5}$ Brian Lau, PhD, ${ }^{1,2,3}$ Hayat Belaid, MD, PhD, ${ }^{1,2,3,4}$ Soledad Navarro, MD, ${ }^{4}$ Marie Vidailhet, MD, PhD, ${ }^{1,2,3,5}$ Eric Bardinet, PhD, 1,2,3,6 Sara Fernandez-Vidal, PhD, , 2,3,6 Marie-Laure Welter, MD, PhD. ${ }^{1,2,3,7}$

1 Université Pierre et Marie Curie-Paris 6, Centre de Recherche de I'Institut du Cerveau et de la Moelle épiniere (CRICM), UMR-S975, Paris, France;

2 INSERM, U975, Paris, France;

3 CNRS, UMR 7225, CR-ICM, Paris, France;

4 Neurosurgery Department, APHP, Hôpitaux Universitaires Pitié-Salpêtrière/Charles Foix, Paris, France;

5 Neurology Department, APHP, Hôpitaux Universitaires Pitié-Salpêtrière/Charles Foix, Paris, France;

6 Centre de Neuroimagerie de Recherche de l'Institut du Cerveau et de la Moelle épinière (CENIR ICM), Paris, France;

7 Neurophysiology Department, CHU Rouen, Normandie University, Rouen, France.

* These authors contributed equally

Correspondence to: ML Welter, Neurophysiology Department, University Hospital Rouen, Rouen-Normandie University, Hôpital Charles Nicolle, 1 rue de Germont, 76032 Cedex, Rouen, France. E-mail: marielaure.welter@icm-institute.org. 
Statistics: Title 139 characters including spaces; Abstract 250 words ; Manuscript 3142 words ; 4 Figures, 34 references. 


\section{Abstract}

Introduction Freezing of gait (FOG) and falls are the most disabling motor symptoms in Parkinson's disease (PD) patients. The effects of subthalamic deep-brain-stimulation (STNDBS) on FOG and falls are still a matter of controversy, and factors contributing to their outcome have yet to be defined.

Methods We examined the relationship between FOG and falls after STN-DBS and preoperative clinical features, MRI voxel-based-morphometry (VBM) analysis and statistical mapping of electrode locations.

Results : 331 patients (age at surgery $=57.7 \pm 8.4$ years ; disease duration $=12.5 \pm 5$ years) were included in the final analysis, with VBM analysis in 151 patients. After surgery, FOG was aggravated in 93 patients and falls in 75 patients. After surgery, FOG severity was related to its level before surgery without dopaminergic treatment, the dopaminergic treatment dosage and severity of motor fluctuations after surgery; and falls severity to lower postoperative cognitive performance. VBM analyses revealed that, relative to other patient groups, patients with FOG worsening had putamen grey matter density decrease, and fallers patients a left postcentral gyrus atrophy. The best effects of STN-DBS on FOG and falls were associated with the location of contacts within the STN, but no specific location related to aggravation.

Conclusions: FOG and falls are reduced after STN-DBS in about $1 / 3$ of patients, with the best effects obtained for electrodes located within the STN. Clinicians should be aware that, after STN-DBS, FOG severity is related to preoperative FOG severity whatever its dopa-sensitivity; and falls to lower postoperative cognitive performance; and atrophy of cortico-subcortical brain areas. 
Key words: Freezing of gait, falls, subthalamic nucleus, deep brain stimulation, Parkinson's disease 


\section{Introduction}

Freezing of gait (FOG) and falls are the most disabling motor symptoms in Parkinson's disease (PD) patients, leading to impaired quality of life and increased morbidity and mortality [1,2]. Fifty to 70\% of PD patients had FOG and/or falls after 10 years of disease duration, that increase in severity with time and are partially and inconsistently improved by the dopaminergic treatment [3]. For more than 20 years, subthalamic deep brain stimulation (STN-DBS) has been recognized as an effective treatment to alleviate PD patients' motor symptoms [4]. On average, pre-existing FOG and/or falls improve significantly after STN-DBS [5]. However, some PD patients show residual or increased FOG and/or falls in the year following surgery $[3,6]$. Some studies, performed in small samples of patients, have suggested that modifying the parameter settings of the STN-DBS may improve the FOG [3]. Furthermore, the factors that contribute to the aggravation of FOG and/or falls after surgery are unknown, in particular at the individual level [7], and no predictive factors for the postoperative outcome of these disabling motor signs have ever been clearly identified.

Our goal was to determine the factors that contribute to FOG and falls outcome after STN-DBS in our large single-centre cohort of 383 PD patients. More specifically, we looked for correlations between FOG and falls severity in the year following STN-DBS and preoperative clinical features, MRI parameters, and the location of the stimulating contacts, to identify patients at risk of developing or aggravating FOG or falls after STN-DBS. 


\section{Methods}

\section{Patients}

Between February 1996 and October 2013, 383 PD patients (mean [SD] age=54.8 [8.8] years) were operated for STN-DBS at the Pitié-Salpêtrière Hospital (Paris, France), as previously described [8]. This study received approval from the local ethics committee.

\section{Neurosurgical procedure}

The brain MRI acquisition including T1 and T2 sequences was performed before surgery to define trajectories and STN targets. Quadripolar electrodes (Medtronic, 3389-28) were implanted, and connected to a pulse generator (Medtronic)[8].

\section{Clinical evaluation}

All patients' assessments were done according to the usual care plan for PD patients operated for STN-DBS in our centre, with assessments three months before and one year after surgery [8].

FOG and falls were assessed using item 14 («FOG») and 13 («falls in the absence of FOG»), respectively, of the Unified Parkinson's Disease Rating Scale (UPDRS) part II, with scores ranging from 0-no sign to 4-severe signs. These scores were obtained by patient interview, and assessed both with (ON-dopa) and without (OFF-dopa) dopaminergic treatment.

Parkinsonian disability was assessed using the UPDRS part I to IV (I :mental status, II : activities of daily living, III : motor disability and IV : levodopa related complications with dyskinesias and motor fluctuations subscores). UPDRS part III (motor disability) was assessed 
in the OFF-dopa (12 hours after antiparkinsonian treatment interruption) and ON-dopa (after administration of a single suprathreshold dose of levodopa) conditions. The severity of axial motor signs was also calculated (sum of the following items: speech, gait, posture, postural instability-items $18,28,29$ and 30, respectively).

Executive functions, attention, memory and visuo-constructive abilities were quantified using the Mattis Dementia Rating Scale (MDRS) and the 'Frontal score' [8]. Depressive symptoms were assessed with the Montgomery-Åsberg Depression Rating Scale (MADRS, depression diagnosis: score $>13$ ).

The levodopa-equivalent dosage and stimulation parameters (pulse width, frequency, intensity) were adjusted to suit the patients' condition.

\section{MRI imaging analysis}

Voxel-based morphometry analysis (VBM) was performed on the preoperative T1images (with no gadolinium) to quantify changes in the grey and white matter concentration ( $n=151$ patients) with the Statistical Parametric Mapping 8 software package (SPM8, Wellcome Trust Centre for Neuroimaging, London, UK). Anatomical locations of the map were identified with the Talairach Client 2.4.2 [9]. Because of our a priori hypothesis, we also used a small volume correction (SVC), comprising the basal ganglia structures and midbrain using two masks.

The electrode location was determined using the postoperative MRI scans or helical tomo-densitometry fused with the preoperative MRI on a 3D histological and deformable basal ganglia atlas. Contact coordinates were measured and normalised in the atlas space 
with reference to the posterior commissure (Absolute laterality: X; Antero-posteriority: $Y$, Depth: Z)[10].

\section{Statistical analysis}

Our main outcome measures were the changes in FOG and falls subscores between baseline and at one year, relative to the same preoperative dopa condition. We classified patients as having FOG or falls if the score on item 14 or 13 was equal to or more than one. The types of FOG and falls were classified following Espay et al. [11]: FOG or falls were considered «OFF», i.e. OFF-FOG or OFF-Falls, if only present without dopaminergic treatment; "ON», i.e ONFOG or ON-Falls, if only present with dopaminergic treatment ; «Resistant», i.e. R-FOG or RFalls, if present both without and with dopaminergic treatment. We considered that patients worsened after STN-DBS if the score increased or improved if it decreased.

To determine which demographic and preoperative motor disability, cognitive status, mood, dopaminergic treatment dosage and related complications contribute to FOG or falls outcome, we performed ANOVA tests to compare preoperative factors between patients groups (increased vs decreased vs no change in FOG or falls severity). The relationship between FOG and falls severity and cognitive status, mood, dopaminergic treatment dosage and related complications after surgery was examined using Pearson correlation tests. A Bonferroni correction was applied and the level of significance was set at p-value $<0.001$ and $<0.0022$, for ANOVA and Pearson tests, respectively. Statistical analyses were performed using the Statview Software package.

For VBM analysis, the SPM8 factorial design specification was used in order to establish the statistical global linear model (GLM) for group comparison. Focal grey and 
white matter volume differences between patients were assessed using Student's t tests. Age was incorporated as a covariate for all analyses and MDRS cognitive score only for falls. The level of statistical significance was set as $p<0.001$ throughout the whole brain and clusters were ultimately considered significant at $p<0.05$, corrected for multiple comparisons using family-wise error (FWE).

The statistical inference linking electrode locations in 3D to its effects on FOG and falls was performed by constructing three 3D maps [12] : 1) the mean effect image, 2) the $t$ image and 3) the P image (see supplementary methods). The mean effect image shows the weighted mean effect at each voxel of the stimulating contact on the outcome measure (FOG or falls). The $t$ image shows a weighted $t$ test at each voxel, taking into account the number of participants contributing data to each voxel. The P image, or probability map, gives the probability of exceeding the corresponding t statistic at each voxel. We implemented a permutation analysis to address the multiple comparison and sample bias problem and obtained a single global $p$ value to test whether DBS effects differ significantly in relation to the location of the stimulating contact. We created 3D images to display statistical maps within the right and left STN and surrounding structures from our 3D basal ganglia atlas [10].

\section{Data statement}

All relevant data are within the paper. Anonymised data will be shared by request from any qualified investigator, at the Brain and Spine Institute, Paris, France. 


\section{Results}

Fifty-two of the 383 PD patients were excluded from the final statistical analysis (Figure 1). We therefore analysed data from 331 PD patients (207 M/124 F; mean [SD] age: 57.7 [8.4] years, mean [SD] disease duration: 12.5 [5.0] years) (see Figure 1 and eTable 1 for details).

\section{Progression of FOG after STN-DBS}

Before surgery, 66 out of 331 PD patients had no FOG (20\%), and 265 patients had preoperative FOG (80\%), with 166 having OFF-FOG (50.5\%) and 99 R-FOG (29.5\%)(Figure 1). One year after surgery, 144 patients were free of FOG (43.5\%), 56 had OFF-FOG (17\%), 125 had R-FOG (37.5\%) and 6 had ON-FOG (2\%)(Figure 1). Among the 265 patients with FOG before surgery, 111 (42\%) were free of FOG after STN-DBS, and among the 66 patients without FOG before surgery, 33 (50\%) developed FOG. Finally, one year after surgery, FOG severity decreased in 122 patients (37\%), was unchanged in 114 (34.5\%) and increased in 95 (28.5\%) (eTable 2). Comparing patients with improved, aggravated or unchanged FOG severity after STN-DBS, we found significant differences in the preoperative falls, FOG, ADL, motor disability and axial scores (OFF and ON-dopa, except falls ON-dopa), with higher scores before surgery in patients with post-operative FOG improvement as compared to other patients (eTable 2).

Correlation analyses revealed that the FOG severity after STN-DBS (OFF and ONdopa) was only positively related to the preoperative FOG severity, in the absence of dopaminergic treatment $(p=0.0004$ and $p=0.0006$, for OFF and ON-dopa, respectively, eTable 4). The postoperative FOG severity OFF-dopa was also positively related to the postoperative severity of motor fluctuations and dopaminergic treatment dosage $(p=0.0003$ and $p=0.002$, 
respectively) and the postoperative FOG severity ON-dopa to the postoperative motor fluctuations severity ( $p=0.0005$ ) (eTable 4). No other significant relationships were found between FOG severity and other pre- or post-operative factors (eTable 4).

In the 151 patients with MRI data included in the VBM analysis, we found no significant difference between PD patients with- versus without- FOG after STN-DBS. Conversely, PD patients that developed or aggravated FOG after STN-DBS, showed significantly reduced bilateral grey matter density within the sensorimotor and associative putamen, and an increase in the left inferior frontal and right inferior temporal gyri, in comparison to PD patients with decreased or unchanged FOG severity (Figure 2A).

No significant difference in stimulating contact coordinates (eFigure 1) or parameter settings (eTable 2) was found between PD patients with- versus without FOG after STN-DBS, with only 16 patients having a stimulation frequency lower than $130 \mathrm{~Hz}$. Statistical maps of stimulating contact locations revealed that the region that was the most strongly associated with FOG improvement was located bilaterally within the centre of the STN, at the junction between the sensorimotor and associative sub-territories (Figure 3). Conversely, no region was found to be associated with FOG aggravation.

\section{Progression of falls after STN-DBS}

Before surgery, 203 out of 331 (61\%) patients were free of falls, 67 patients had OFF-Falls (21.5\%), 61 had R-Falls (17.5\%) and none had ON-Falls (Figure 1). One year after surgery, 224 patients were free of falls (40\%), 23 had OFF-Falls (13.5\%), 75 had R-Falls (40\%) and 9 had ON-Falls (2\%)(Figure 1). Among the 128 patients with falls before surgery, 50 (39\%) were free of falls after STN-DBS, and among the 203 patients without falls before surgery, 57 
(28\%) developed falls. Finally, one year after surgery, falls severity decreased in 83 patients (25\%), was unchanged in 173 (52.5\%) and increased in 75 (22.5\%)(eTable 3). Comparing patients with improved, aggravated or unchanged falls severity, we found significant differences in the preoperative falls, FOG, ADL, motor disability and axial scores (OFF and ON-dopa, except FOG and ADL ON-dopa) between groups, with higher scores in patients with post-operative fall improvement as compared to other patients (eTable 3).

Correlation analyses revealed that the postoperative falls severity (OFF and ON-dopa) was only positively related to the preoperative ADL score ON-dopa $\left(p<10^{-4}\right.$, for OFF and ONdopa) and negatively to the postoperative cognitive status (MDRS, $p=0.0005$ and $p<10^{-4}$, for OFF and ON-dopa, respectively)(eTable 4).

In the 151 patients included in the VBM analysis, we found a significant decrease in grey matter density in the left postcentral gyrus and an increase in the culmen of the right cerebellar lobe in PD patients with falls after surgery, relative to non-falling PD patients (Figure 2B). No significant difference was found between patients who had an improvement versus an aggravation of falls after STN-DBS.

No significant difference in stimulating contact coordinates (eFlgure 1) or parameter settings (eTable 3 ) were found between PD patients with- versus without- falls after STNDBS. Statistical maps of stimulating contact locations revealed that the region that was the most strongly associated with the improvement of falls was located within the STN, in particular in its more dorsal and medial part near the zona incerta, and also in a ventral STN region near the substantia nigra pars reticulata (SNr, Figure 4). Conversely, no region was found to be associated with worsening of falls. 


\section{Discussion}

The main findings of our study are that FOG and falls are improved by STN-DBS in about $1 / 3$ of patients. Patients with more severe symptoms are more likely to improve after STN-DBS, and, conversely, patients with no or mild symptoms tend to remain stable or worsen. The presence of FOG or falls after surgery does not seem directly associated with the presence of FOG or falls before surgery, respectively. However, the severity of FOG after surgery is mainly related to the severity of FOG before surgery, independently of the patient's dopasensitivity, and the severity of falls to lower postoperative cognitive performance. The anatomical study revealed that post-operative FOG is related to putamen atrophy and falls to left postcentral gyrus atrophy. The best effects of STN-DBS on FOG and falls were obtained with stimulating contacts located within the STN area, with no specific location related to worsening.

\section{STN-DBS and FOG}

In our study, FOG severity decreased sas significantly improved in $37 \%$ of patients after STN-DBS, as reported in smaller cohorts $[5,7,13]$. We found that the severity of FOG after STN-DBS only correlated with the severity of preoperative FOG, independently of its improvement with dopaminergic treatment, conversely to that reported for akinesia, rigidity or tremor [14], but also for FOG [7]. This and the decreased putamen grey matter density found in our patients suggest that postoperative FOG is mainly related to the natural progression of the disease with additional striatal lesions $[3,15]$, also reported in advanced PD patients [16]. A decreased caudate nucleus activity during motor imagery of leg movements or gait in 'freezer' PD patients $[17,18]$ and the occurrence of FOG after bilateral 
putamen lesions [19] in non-parkinsonian patients also support this hypothesis. High grey matter density in the left inferior frontal and right inferior temporal gyri was also found in our patients with worsened post-operative FOG. The change in the inferior temporal gyrus, that receives connections from the visual processing areas, could be related to the increased visual dependence of gait in 'freezer' patients [20], with parieto-temporo-occipital sensory associative cortices increased metabolic activity previously related to FOG improvement after STN-DBS [21]. Finally, these morphological and functional changes in associative cortical regions may reflect a compensatory mechanism to facilitate correct multisensory information processing [18,22]. FOG after STN-DBS was also correlated with postoperative dopaminergic treatment dosage and severity of motor fluctuations. This could indicate that FOG patients had received higher dopaminergic doses to improve FOG, leading to more severe motor fluctuations, as previously reported [23]. The best location for FOG improvement was the centre of the STN, i.e. at the junction of the sensorimotor and associative parts of the STN [24], suggesting that stimulation applied in this STN area may promote better simultaneous processing of sensorimotor and associative information [25], therefore leading to better effects on FOG.

\section{STN-DBS and falls}

After STN-DBS, falls severity decreased improvedin $25 \%$ of patients, and no specific preoperative factor related to the falls outcome. The severity of falls after STN-DBS was only linked to lower postoperative cognitive performance. A relationship between falls and mild cognitive impairment has been previously reported in PD patients [26], suggesting that patients prone to falling suffered from brain dysfunction in areas involved in both balance 
control and cognitive functions. Our falling PD patients had indeed significant grey matter density decrease of the left postcentral gyrus, a region highly involved in the integration of sensory and proprioceptive information and vertical perception and shown to be impaired in relation to postural instability in PD patients $[27,28]$; and grey matter increase of the right cerebellar lobe, a region shown to be overactive during imagined walking with obstacle negotiation in PD patients [29]. We found no significant changes in grey and white matter density of the basal ganglia or the mesencephalic locomotor region (MLR), which have been previously reported to be atrophic or dysfunctional in PD patients with falls $[17,30]$ but also in other cognitive areas. However, our PD patients had a very good levodopa responsive form of the disease and no cognitive deficit (mean MDRS>130). Finally, improvements in falls were found to be related to stimulating contact locations within two sites of the STN: one deep near the SNr and one more dorsal and medial near the zona incerta. Interestingly, in small cohort studies, SNr stimulation has been shown to improve postural control and/or gait of PD patients when applied solely [31], or interleaved with the STN [32]; and zona incerta DBS decreased axial subscores when applied concomitantly with MLR stimulation [33].

\section{Limitations}

One limitation of our study is the FOG and falls assessment using items 14 and 13 of the UPDRS part II. Unfortunately, the FOG questionnaire (FOG-Q) was validated in 2009 , and therefore not used in our patients operated from 1996. However, Item 14 of the UPDRS II has been shown to be highly correlated with the FOG-Q to detect patients with FOG [34]. To our knowledge, no specific questionnaire has been validated to assess fall severity and a fall 
diary could have given more details about fall occurrence but such an assessment may be considered too subjective with a high potential for missing data. We used two different methods to determine contact locations, one with postoperative MRI ( $n=174$ patients) and the other with CT scans ( $n=157$ patients). This could have introduced a bias between patients for contact location estimation. However, the sub-millimetric differences found between these two methods (eTable 5) does not favour this hypothesis, as well as the rather homogeneous stimulating contact locations. This homogeneous location, predominantly in the centre of the STN [8], prevented us from examining the effects of stimulation applied in other parts of the STN and surrounding structures. However, the use of statistical mapping techniques on the effects of STN-DBS in our large number of patients allowed us to explore the role of the stimulating contact location. Finally, the absence of a control group of nonoperated PD patients or use of functional imaging study prevented us from specifically assessing the role of the disease's natural progression on the postoperative outcome.

\section{Conclusions}

Overall, STN-DBS decreases FOG and falls severity in a substantial proportion of PD patients. The best location is the STN itself, with no specific location associated with post-operative worsening, suggesting that STN-DBS does not aggravate FOG and falls per se. Neurologists should be aware that preoperative FOG severity, whatever its dopa-sensitivity, and low postoperative MDRS score are predictive of residual postoperative FOG and falls, respectively.

Our results also highlight the potential anatomical dysfunction of the putamen and orbitofrontal and temporal cortices in FOG, and of the associative cortical regions and 
cerebellum in falls. Specific assessments of gait and balance abilities, in relationship with stimulation condition, and additional imaging using brain connectivity methods and neuropathological studies, are now needed to further understand FOG and falls outcomes after STN-DBS. 


\section{References}

[1] Kempster PA, O'Sullivan SS, Holton JL, Revesz T, Lees AJ. Relationships between age and late progression of Parkinson's disease: a clinico-pathological study. Brain. 2010;133:175562.

[2] Bloem BR, van Vugt JP, Beckley DJ. Postural instability and falls in Parkinson's disease. Adv Neurol. 2001;87:209-23.

[3] Fasano A, Aquino CC, Krauss JK, Honey CR, Bloem BR. Axial disability and deep brain stimulation in patients with Parkinson disease. Nat Rev Neurol. 2015;11:98-110.

[4] Limousin P, Krack P, Pollak P, Benazzouz A, Ardouin C, Hoffmann D, et al. Electrical stimulation of the subthalamic nucleus in advanced Parkinson's disease. N Engl J Med. 1998;339:1105-11.

[5] Ferraye MU, Debu B, Fraix V, Xie-Brustolin J, Chabardes S, Krack P, et al. Effects of subthalamic nucleus stimulation and levodopa on freezing of gait in Parkinson disease. Neurology. 2008;70:1431-7.

[6] Stolze H, Klebe S, Poepping M, Lorenz D, Herzog J, Hamel W, et al. Effects of bilateral subthalamic nucleus stimulation on parkinsonian gait. Neurology. 2001;57:144-6.

[7] Ferraye MU, Ardouin C, Lhommee E, Fraix V, Krack P, Chabardes S, et al. Levodoparesistant freezing of gait and executive dysfunction in Parkinson's disease. Eur Neurol. 2013;69:281-8.

[8] Welter ML, Schupbach M, Czernecki V, Karachi C, Fernandez-Vidal S, Golmard JL, et al. Optimal target localization for subthalamic stimulation in patients with Parkinson disease. Neurology. 2014;82:1352-61. 
[9] Lancaster JL, Woldorff MG, Parsons LM, Liotti M, Freitas CS, Rainey L, et al. Automated Talairach atlas labels for functional brain mapping. Hum Brain Mapp. 2000;10:120-31. [10] Bardinet E, Bhattacharjee M, Dormont D, Pidoux B, Malandain G, Schupbach M, et al. A three-dimensional histological atlas of the human basal ganglia. II. Atlas deformation strategy and evaluation in deep brain stimulation for Parkinson disease. J Neurosurg. 2009;110:208-19.

[11] Espay AJ, Fasano A, van Nuenen BF, Payne MM, Snijders AH, Bloem BR. "On" state freezing of gait in Parkinson disease: a paradoxical levodopa-induced complication. Neurology. 2012;78:454-7.

[12] Eisenstein SA, Koller JM, Black KD, Campbell MC, Lugar HM, Ushe M, et al. Functional anatomy of subthalamic nucleus stimulation in Parkinson disease. Ann Neurol. 2014;76:27995.

[13] Vercruysse S, Vandenberghe W, Munks L, Nuttin B, Devos H, Nieuwboer A. Effects of deep brain stimulation of the subthalamic nucleus on freezing of gait in Parkinson's disease: a prospective controlled study. J Neurol Neurosurg Psychiatry. 2014;85:871-7.

[14] Welter ML, Houeto JL, Tezenas du Montcel S, Mesnage V, Bonnet AM, Pillon B, et al. Clinical predictive factors of subthalamic stimulation in Parkinson's disease. Brain. 2002;125:575-83.

[15] Nutt JG, Bloem BR, Giladi N, Hallett M, Horak FB, Nieuwboer A. Freezing of gait: moving forward on a mysterious clinical phenomenon. Lancet Neurol. 2011;10:734-44.

[16] McNeill TH, Brown SA, Rafols JA, Shoulson I. Atrophy of medium spiny I striatal dendrites in advanced Parkinson's disease. Brain Res. 1988;455:148-52. 
[17] Snijders AH, Leunissen I, Bakker M, Overeem S, Helmich RC, Bloem BR, et al. Gaitrelated cerebral alterations in patients with Parkinson's disease with freezing of gait. Brain. 2011;134:59-72.

[18] Shine JM, Matar E, Ward PB, Bolitho SJ, Gilat M, Pearson M, et al. Exploring the cortical and subcortical functional magnetic resonance imaging changes associated with freezing in Parkinson's disease. Brain. 2013;136:1204-15.

[19] Imai H. Clinicophysiological features of akinesia. Eur Neurol. 1996;36:9-12.

[20] Azulay JP, Mesure S, Amblard B, Pouget J. Increased visual dependence in Parkinson's disease. Perceptual and motor skills. 2002;95:1106-14.

[21] Lyoo CH, Aalto S, Rinne JO, Lee KO, Oh SH, Chang JW, et al. Different cerebral cortical areas influence the effect of subthalamic nucleus stimulation on parkinsonian motor deficits and freezing of gait. Mov Disord. 2007;22:2176-82.

[22] Bartels AL, Leenders KL. Brain imaging in patients with freezing of gait. Mov Disord. 2008;23 Suppl 2:S461-7.

[23] Amboni M, Stocchi F, Abbruzzese G, Morgante L, Onofrj M, Ruggieri S, et al. Prevalence and associated features of self-reported freezing of gait in Parkinson disease: The DEEP FOG study. Parkinsonism Relat Disord. 2015;21:644-9.

[24] Grabli D, McCairn K, Hirsch EC, Agid Y, Feger J, Francois C, et al. Behavioural disorders induced by external globus pallidus dysfunction in primates: I. Behavioural study. Brain. 2004;127:2039-54.

[25] Mallet L, Schupbach M, N'Diaye K, Remy P, Bardinet E, Czernecki V, et al. Stimulation of subterritories of the subthalamic nucleus reveals its role in the integration of the emotional and motor aspects of behavior. Proc Natl Acad Sci U S A. 2007;104:10661-6. 
[26] Fasano A, Romito LM, Daniele A, Piano C, Zinno M, Bentivoglio AR, et al. Motor and cognitive outcome in patients with Parkinson's disease 8 years after subthalamic implants. Brain. 2010;133:2664-76.

[27] Vaugoyeau M, Viel S, Assaiante C, Amblard B, Azulay JP. Impaired vertical postural control and proprioceptive integration deficits in Parkinson's disease. Neuroscience. 2007;146:852-63.

[28] Pereira CB, Kanashiro AK, Maia FM, Barbosa ER. Correlation of impaired subjective visual vertical and postural instability in Parkinson's disease. J Neurol Sci. 2014;346:60-5. [29] Maidan I, Rosenberg-Katz K, Jacob Y, Giladi N, Deutsch JE, Hausdorff JM, et al. Altered brain activation in complex walking conditions in patients with Parkinson's disease. Parkinsonism Relat Disord. 2016;25:91-6.

[30] Müller ML, Albin RL, Kotagal V, Koeppe RA, Scott PJ, Frey KA, et al. Thalamic cholinergic innervation and postural sensory integration function in Parkinson's disease. Brain. 2013;Nov;136:3282-9.

[31] Chastan N, Westby GW, Yelnik J, Bardinet E, Do MC, Agid Y, et al. Effects of nigral stimulation on locomotion and postural stability in patients with Parkinson's disease. Brain. 2009;132:172-84.

[32] Weiss D, Walach M, Meisner C, Fritz M, Scholten M, Breit S, et al. Nigral stimulation for resistant axial motor impairment in Parkinson's disease? A randomized controlled trial. Brain. 2013;136:2098-108.

[33] Khan S, Javed S, Mooney L, White P, Plaha P, Whone A, et al. Clinical outcomes from bilateral versus unilateral stimulation of the pedunculopontine nucleus with and without 
concomitant caudal zona incerta region stimulation in Parkinson's disease. Br J Neurosurg. 2012;26:722-5.

[34] Giladi N, Tal J, Azulay T, Rascol O, Brooks DJ, Melamed E, et al. Validation of the freezing of gait questionnaire in patients with Parkinson's disease. Mov Disord. 2009;24:655-61. 


\section{Figure legends}

Figure 1. FOG and falls in 331 PD patients before, and one year after, STN-DBS

Figure 2. Relationship between the effects of STN DBS on FOG and falls and preoperative brain anatomy in PD patients

A) Brain voxel-based morphometry analysis in PD patients with increased FOG severity after STN-DBS as compared to PD patients with decreased or no change in FOG severity. Statistical parametric map of reduced (upper) and increased (lower) grey matter density superimposed on the MNI152 template with sagittal, frontal and axial views. B) Brain voxel-based morphometry analysis in PD patients presenting falls after STN-DBS as compared to PD patients who did not. Statistical parametric map of reduced (upper) and increased (lower) grey matter superimposed on the MNI152 template with sagittal, frontal and axial views.

\section{Figure 3. STN-DBS effects on freezing of gait in PD patients.}

Statistical images in colour, superimposed on brain MRI coronal slices and centred on the left (upper) and right (lower) STN in the 3D atlas. Upper panels for each side show weighted mean effect images (thresholded at $n \geq 6$ ) and lower panels show $p$ images (thresholded at $p$ $\leq 0.01$ ) obtained each $\mathrm{mm}$ from 13 (left) to 8 (right) $\mathrm{mm}$ anterior to the posterior commissure. For the mean effect images, an increased FOG severity after surgery is represented by a positive score (red) and a decrease by a negative score (green-blue). Structures are shown superimposed on the brain MRI with the thalamus (Thal, green outline), zona incerta (ZI, brown outline), red nucleus (RN, red outline), substantia nigra pars 
reticulata (SNr, black outline) and the subthalamic nucleus with its sensorimotor (green outline), associative (pink outline) and limbic (yellow outline) subterritories.

\section{Figure 4. STN-DBS effects on falls in PD patients.}

Statistical images in colour, superimposed on brain MRI coronal slices and centred on the left (upper) and right (lower) STN in the 3D atlas. Upper panels for each side show weighted mean effect images (thresholded at $n \geq 6$ ) and lower panels $p$ images (thresholded at $\mathrm{p} \leq 0.01$ ) obtained each $\mathrm{mm}$ from 13 (left) to 8 (right) $\mathrm{mm}$ anterior to the posterior commissure. For the mean effect images, an increase in falls severity after surgery is represented by a positive score (red) and a decrease by a negative score (green-blue). Structures are shown superimposed on the brain MRI with the thalamus (Thal, green outline), zona incerta (ZI, brown outline), red nucleus (RN, red outline), substantia nigra pars reticulata (SNr, black outline) and the subthalamic nucleus with its sensorimotor (green outline), associative (pink outline) and limbic (yellow outline) subterritories. 


\section{Acknowledgments}

We extend our deepest thanks to all the patients who participated in this research with great dedication. We thank Kevin J. Black (Washington University in St Louis, St Louis, USA) and Lydia Yahia Cherif (CENIR ICM, Paris, France) for their help with the statistical analysis. We thank Fanny Pineau for neuropsychological assessments.

\section{Study Funding}

This study was supported by the Institut National de la Recherche Médicale (INSERM), the 'Institut du Cerveau et de la Moelle Epinière' (ICM) Foundation, and the programme 'Investissements d'avenir' (ANR-10-IAIHU-06).

\section{Authors contributions}

MLW and CK were responsible for the conception and design of the study. MLW obtained funding for the study. CK, FDC, HB, SN, BL, MV, DG, MC and MLW performed clinical investigations. MLW and DG performed parameter adjustments for STN-DBS. FDC, HB, EB and SFV performed the imaging data acquisition and processing. CK, FDC and MLW drafted the report. FDC, CK, DG, HB, BL, MV, EB, SFV and MLW provided substantial input to the first draft. MLW and EB created the figures. All authors reviewed the manuscript and give their final approval of the submitted version. The study was supervised by MLW.

\section{Financial disclosure/Conflicts of interest}

C Karachi, F Dequaire-Cormier, H Belaid, B Lau, M Vidailhet, S Fernandez-Vidal have no financial interests to disclose. 
D Grabli received lecture fees and travel grants from AbbVle, Zambon and Merz. He received grants from the Direction Générale de I'Organisation des Soins (DGOS).

E Bardinet received a research grant from Medtronic, the Michael J. Fox Foundation for Parkinson Research, and the Fondation pour la Recherche Médicale (FRM, France).

ML Welter received research grants from the 'Institut du Cerveau de la Moelle épinière' (ICM) Foundation, the Agence Nationale de la Recherche, the Michael J Fox Foundation for Parkinson Research, and consulting fees from Medtronic. 
A
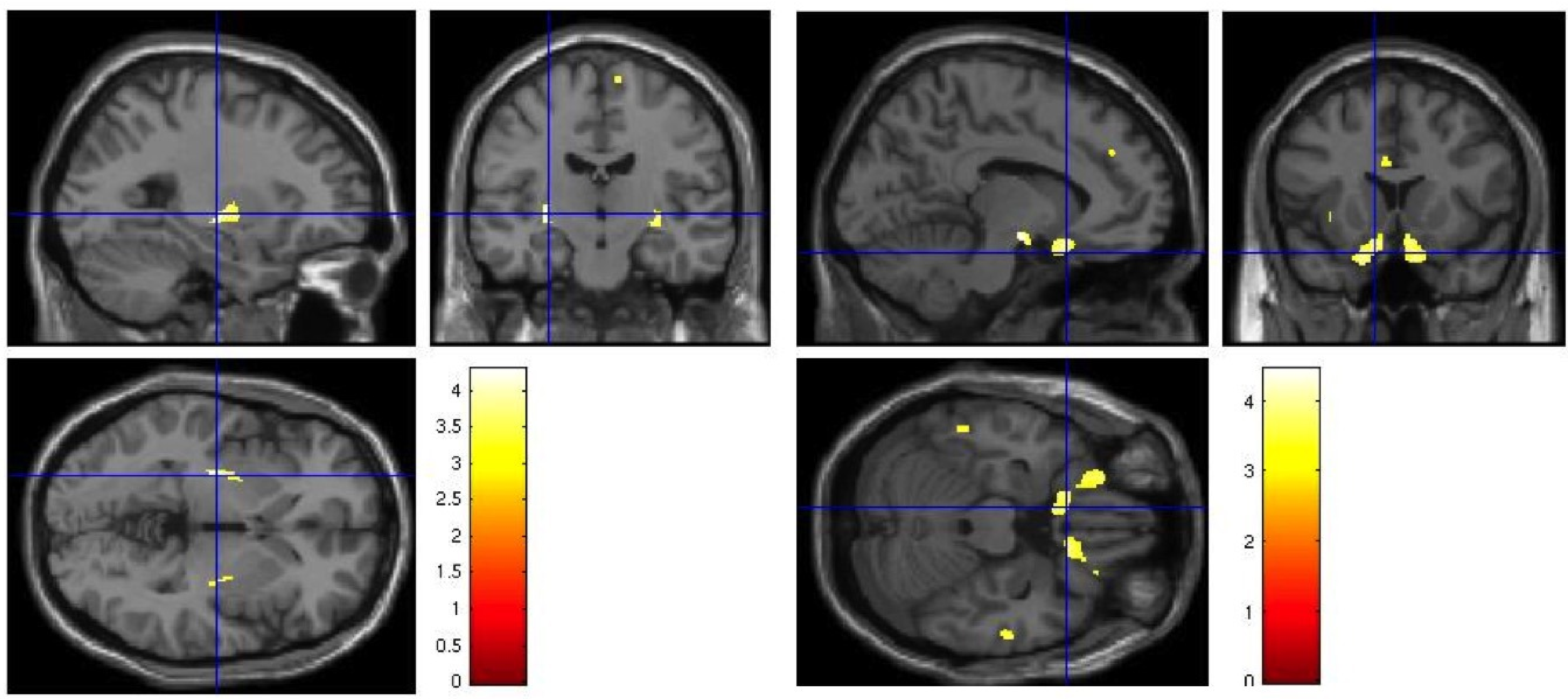

B
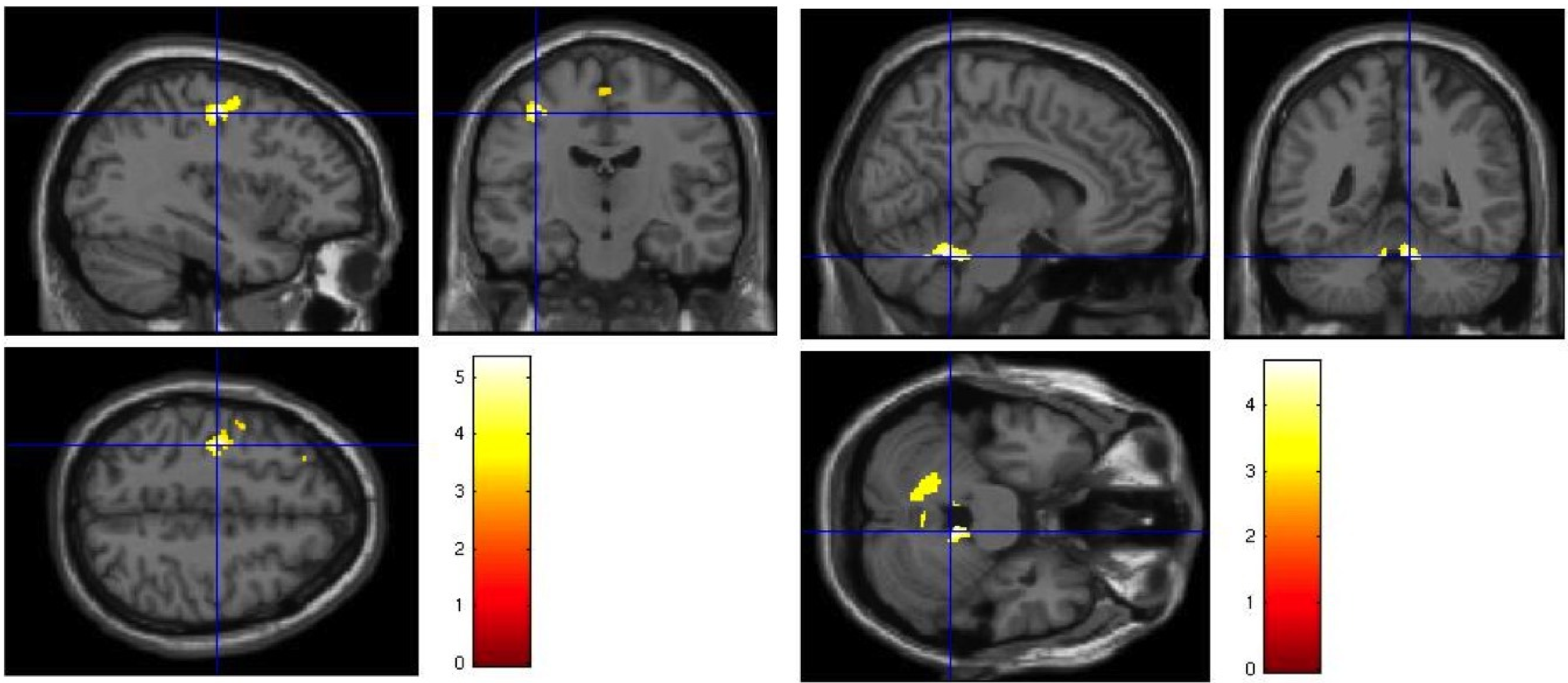


\section{Left side}

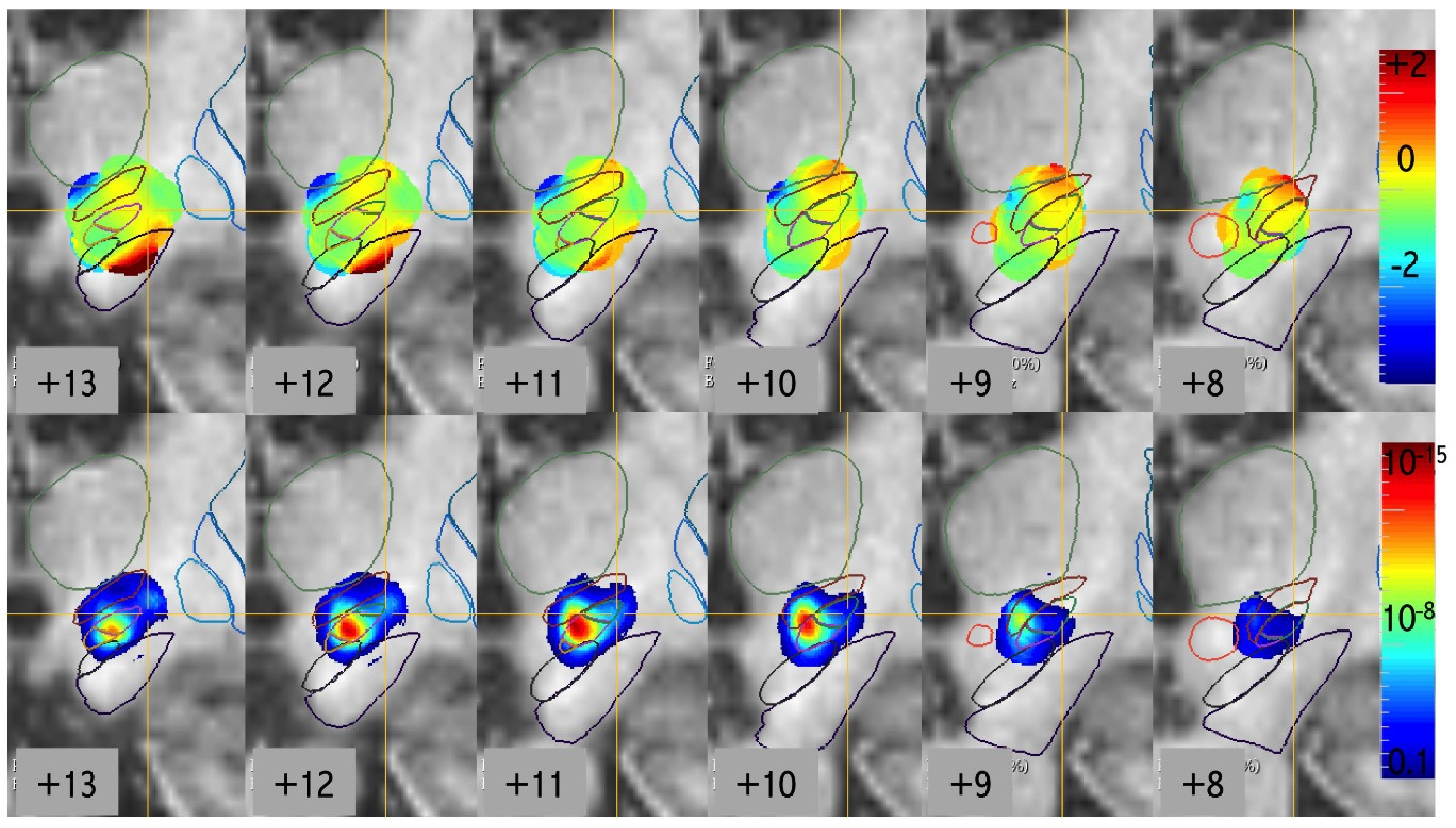

\section{Right side}

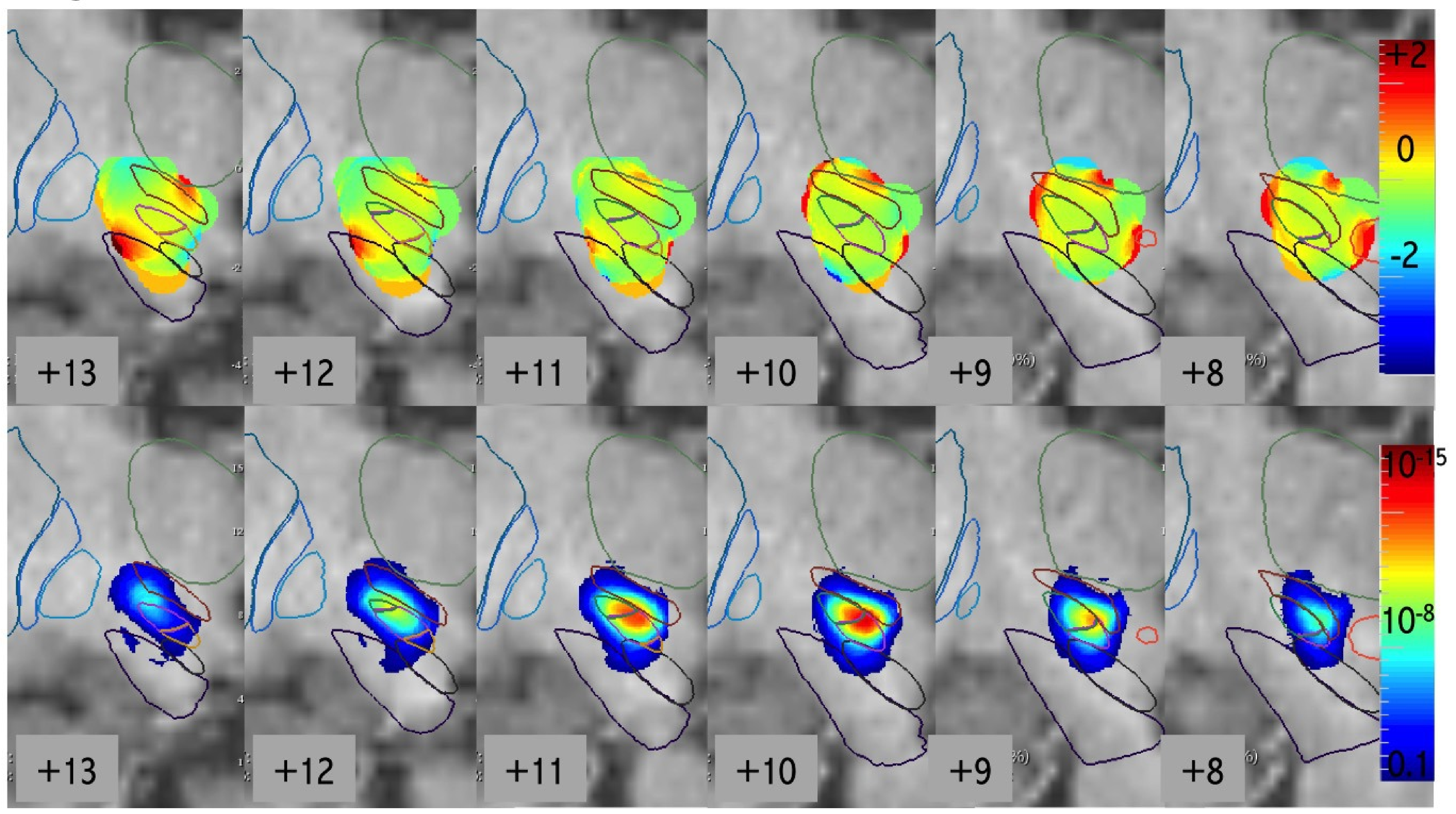




\section{Left side}

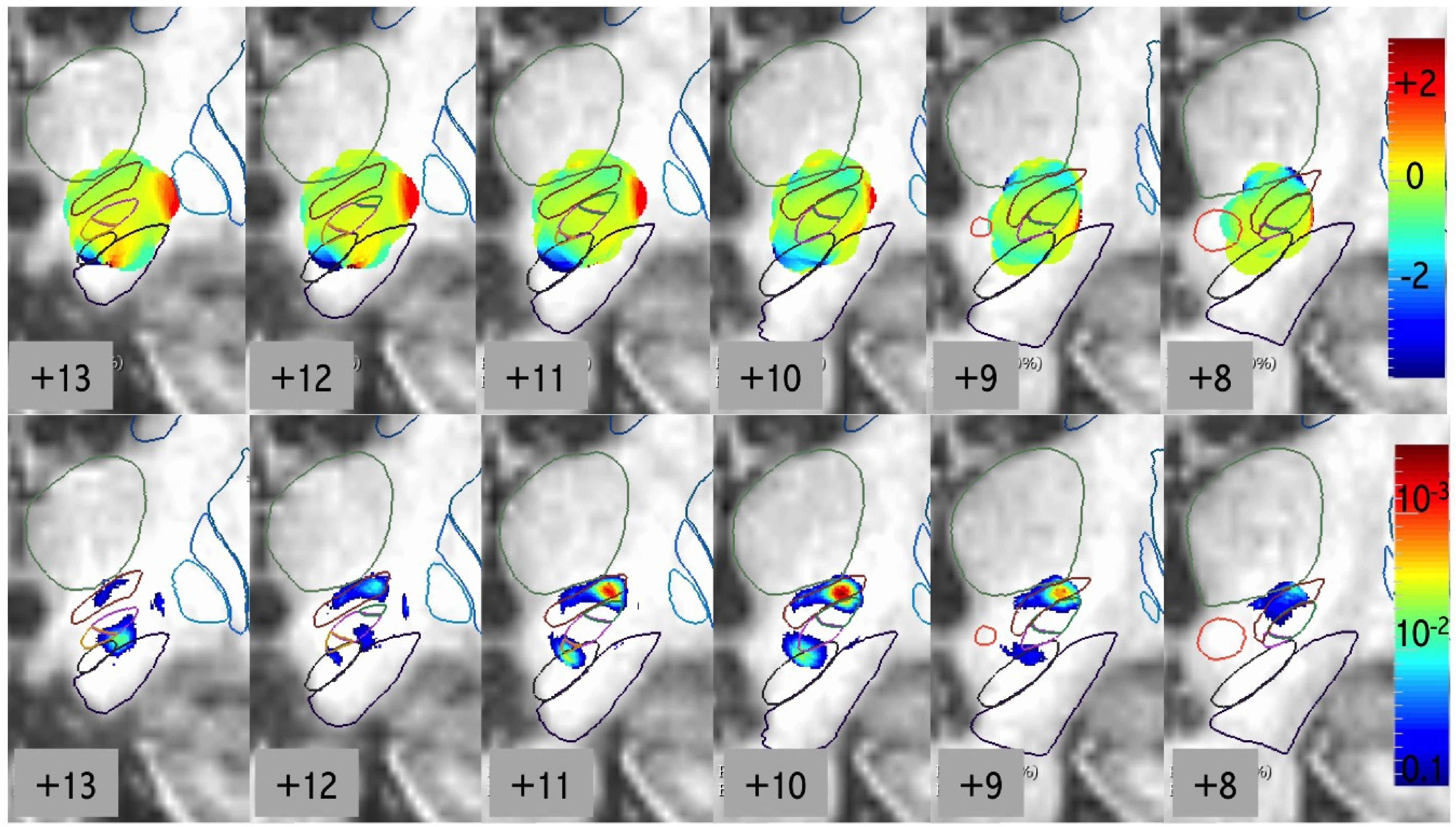

\section{Right side}

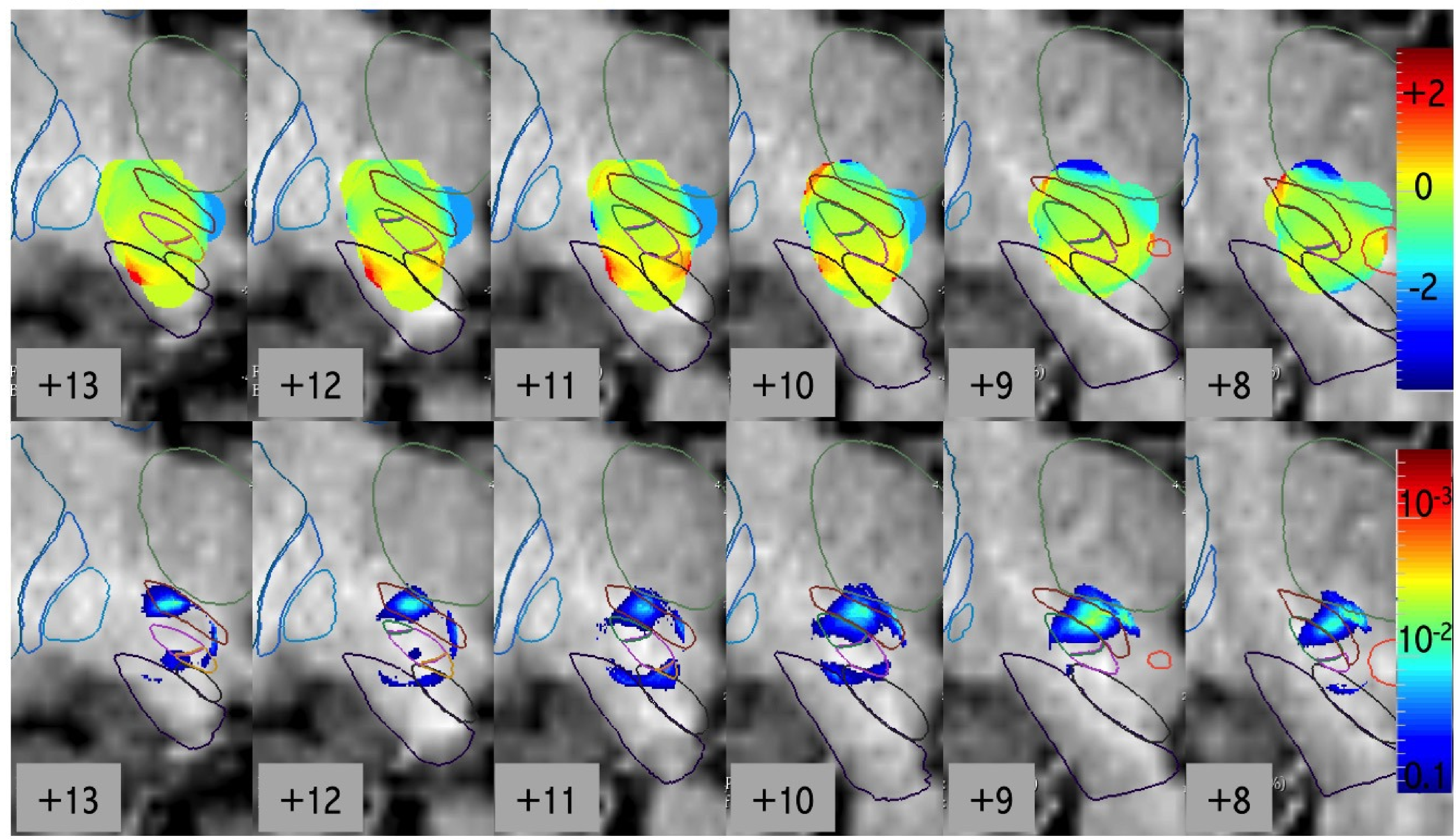

